\title{
2MoTac: Simulation of Button Click by Superposition of Two Ultrasonic Plate Waves
}

\author{
Pierre Garcia ${ }^{1,2(\bowtie)}$, Frédéric Giraud ${ }^{1}$, Betty Lemaire-Semail ${ }^{1}$, \\ Matthieu Rupin' ${ }^{2}$, and Michel Amberg ${ }^{1}$ \\ 1 Univ. Lille, Centrale Lille, Arts Et Metiers Institute of Technology, Yncrea \\ Hauts-de France, ULR 2697 L2EP, 59000 Lille, France \\ \{pierre.garcia,frederic.giraud, \\ betty. lemaire-semail, michel . amberg\}@univ-lille.fr \\ ${ }^{2}$ Hap2u, 20 Rue du Tour de l'Eau, 38400 Saint-Martin-d'Hères, France \\ \{pierre.garcia,matthieu.rupin\}@hap2u.net
}

\begin{abstract}
Recent studies have shown that a button click sensation could be simulated thanks to ultrasonic vibrations. In this context, a travelling wave may enhance the simulation because it creates internal lateral stresses that are released during the stimulation. However, this solution is difficult to integrate on plates. We present 2 MoTac, a method which superpose a longitudinal and a bending mode simultaneously on a plate, in order to create a pseudo-travelling wave. We present the design, and a psychophysical study to deduce the optimal ratio between the bending and longitudinal mode amplitudes, in terms of detection threshold and robustness.
\end{abstract}

Keywords: Haptic display · Tactile perception · Ultrasonic vibration $\cdot$ Vibration mode $\cdot$ Keyclick $\cdot$ Button click

\section{Introduction}

Most of new human-machine interaction devices now rely on touch screens. They have become so cost effective that physical buttons and knobs are removed and replaced by virtual buttons displayed on the flat and hard surface of the display panel in vending machines, car dashboards, and so on. However, touch screens do not involve the sense of touch in the interaction with the machine: they do not provide information through this channel, unlike physical buttons for instance. Therefore, sight is predominant for whom wants to interact with the aforementioned machines, leading to many disadvantages: elderly and visuallyimpaired individuals struggle to use touch screens, and drivers must look away from the road, which is a major safety issue in automobile. Therefore, technological improvements to touch screens are needed, in order to involve touch when interacting with a machine through its touch screen. 
To create the illusion of touching a button on a panel, lateral displacement [1] or vibrotactile stimulation [11] can be used. The low frequency vibration of the touch screen directly stimulates the skin mechanoreceptors; because this vibration is difficult to contain, [3] identifies the frequency response of the touch screen $(\mathrm{FRF})$ and then uses the frequency that maximises the vibration displacement at a specific point. In [7], the authors invert the FRF and can control the vibration at three different positions where actuators are located. However, using vibrotactile stimulation to create the simulation of a button click has the disadvantage to produce audible noise. To cope with this issue, [10] uses the rapid vibration of a standing wave at ultrasonic frequency, to reduce the internal stresses inside the finger pulp due to the friction that appears when pressing the touchscreen. More robust results are obtained if a net tangential force is produced, by using electroadhesion force synchronized on ultrasonic lateral displacement [15] or by using a travelling wave [6]. These solutions however seem difficult to be integrated on plates, without obstructing the view by actuators.

This paper introduces a new concept of tactile stimulation that uses two orthogonal modes to simulate a button click. The elliptical motion of the particles in contact with the fingertip creates a lateral force as if a travelling wave was used. After presenting the technological principle that derives from [2], a study has been conducted in order to define the ratio between the two modes that optimizes the feeling of a single click when reversing the lateral force direction.

\section{Presentation of the Two Modes Stimulation}

\subsection{Theoretical Background}

We consider a plate, which length is $L$, thickness $b$ and height $H$. Its vibration can result in the superposition of different types of modes: the longitudinal modes are characterized by the out-of plane translation of the plate's cross-sections while bending modes are characterized by an in-plane translation and an out-of plane rotation of the cross-section, as described in Fig. 1.

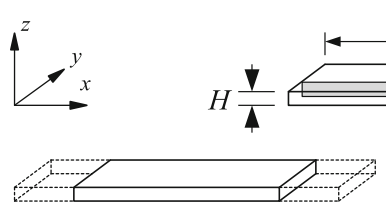

a)

\section{$L$}
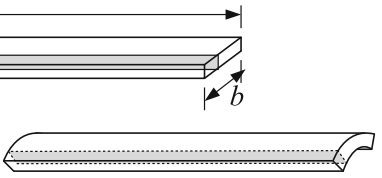

b)

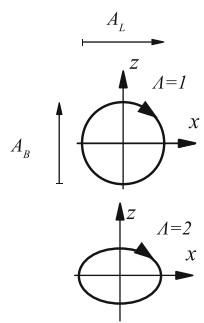

c)

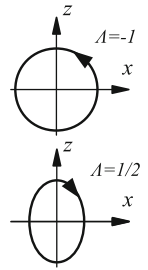

Fig. 1. Vibration mode a) Longitudinal, b) bending and c) Elliptical motion in the middle of the top surface for several values of $\Lambda$ 
When the two modes are produced at the same vibrating frequency, then the point in the middle of the top surface undergoes a displacement which has two components:

- along $x$, due to the longitudinal mode, denoted $a_{L}(t)$,

- along $z$, due to the bending mode, denoted $a_{B}(t)$.

In this paper, the two modes are energized at the same frequency $f$, with a phase shift of $\pm \pi / 2$. We then write:

$$
a_{L}(t)=\frac{A_{L}}{2} \sin (2 \pi f t) \quad a_{B}(t)=\frac{A_{B}}{2} \cos (2 \pi f t)
$$

with $A_{L}$ and $A_{B}$ the vibration amplitude of each mode (peak-peak); if $A_{B}<0$, the phase shift is $\pi / 2$. Hence, by combining the two vibration modes, the points at the top surface follow an elliptical motion, which is able to create a net lateral force, as described in [4]. By changing the ratio $\Lambda=\frac{A_{L}}{A_{B}}$, the shape of the elliptical trajectory is modified, as described in Fig. 1c), promoting the normal displacement over the longitudinal one for $\Lambda<1$ and vice versa for $\Lambda>1$. In the remaining of this paper, we introduce the maximum vibration speed $U_{L}=2 \pi f A_{L}$ and $U_{B}=2 \pi f A_{B}$ because they combine frequency and displacement.

\subsection{Related Work}

To produce a button click sensation, [10] uses a rapid change in friction between the fingertip and a plate by increasing or decreasing ultrasonic vibration. The detection threshold of the click is found to be lower if the friction is decreased compared with a friction increased. A fraction of the elastic energy stored in the finger pulp is due to some tangential contact forces that appear during touch. When it is suddenly released, it creates a stimulus that is detectable by the skin's mechanoreceptors. However, the detection thresholds of this click are higher compared with those measured to detect a friction change in presence of a lateral motion of the fingertip [12]. Therefore, [6] suggests to use a travelling wave instead of a standing wave. Indeed, the results show a lower perceptual threshold for travelling waves, and participants press less times per trial and exert smaller normal force on the surface in order to perceive click effect.

Travelling wave can increase the tangential contact forces due to the elliptical motion of the particles that are in contact with the fingertip, thus increasing the stimulus when they are released. Two theories can explain this phenomenon. In [14], the tangential force derives from the contact conditions between a vibrating plate and an elastic medium. In [9], the authors introduce the lateral viscous forces that are generated by a travelling wave, a phenomenon which is compatible with the existence of the squeeze-film. For both theories, the lateral force is a complex function of the normal and tangential displacements of the particles.

In a travelling wave, the ratio $\Lambda$ between the normal and lateral vibration amplitude is fixed by the geometry of the vibrating plate. To cope for this issue, 
[2] presents a prototype that can produce a longitudinal and a bending mode which resonant frequencies are equal. However, the actuator for the longitudinal mode is bulky, while the vibration amplitude of each mode are not controlled. With 2MoTact, the two components $U_{B}$ and $U_{L}$ can be independently set, offering the possibility to optimize the tuning of $\Lambda$ in order to improve the lateral contact forces produced to enhance the click sensation. A study is then conducted in order to determine which ratio is optimal in terms of psychophysical threshold. To compare the thresholds between each other, the stimulation level is defined as $U=\sqrt{U_{L}^{2}+U_{B}^{2}}$, which combines the vibration speed for the longitudinal and the bending mode. We include in the study the case of a pure bending mode displacement $\left(U_{L}=0, \Lambda=0\right)$.

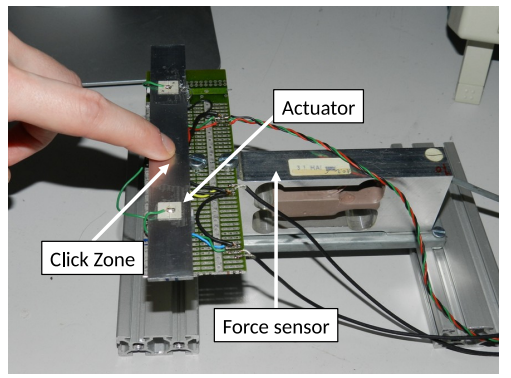

a)

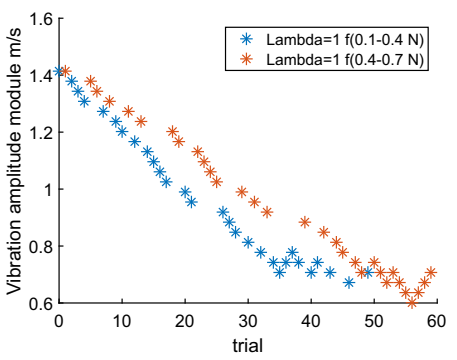

b)

Fig. 2. The Experimental setup a) and typical example of 2 interlaced psychophysical staircases, which targets the $50 \%$ perceptual threshold by a one-up one-down test. At each trial, the implemented staircase is chosen at random with a probability of $1 / 2$.

\subsection{Design of the Plate}

Our prototype consists of a $148 \times 18 \times 2 \mathrm{~mm}^{3}$ aluminium plate, actuated by nine piezoelectric ceramic actuators $(5 \mathrm{~mm} \times 7 \mathrm{~mm} \times 0.5 \mathrm{~mm}$ from Noliac, Denmark) as presented Fig. 3c). The plate was designed such that both modes (longitudinal and bending) can be excited at the same frequency $(f=34 \mathrm{kHz})$. To energize the longitudinal mode, eight actuators placed on the upper and lower side of the plate are used. For the bending mode, only one ceramic placed in the middle of the plate on the lower side is used. Two other ceramic plates $(14 \mathrm{~mm} \times 2 \mathrm{~mm} \times$ $0.3 \mathrm{~mm}$, Noliac Denmark) are added as sensors. Two voltage amplifiers (WMA300 from Falco, The Netherlands) can apply a voltage up to $300 \mathrm{~V}$ peak to peak to the actuators' terminals.

The deformation shape for each mode is presented in Fig. 3. Due to the deformation shape for each mode, the ratio $\Lambda$ is not constant over the top surface, and its value is specified in the middle of the plate. The speed and phase of the 
vibration of each mode are controlled independently, using the vector control method $[4,5]$. From the vector control method we can define:

$$
U_{L}=\sqrt{U_{L d}^{2}+U_{L q}^{2}} \quad U_{B}=\sqrt{U_{B d}^{2}+U_{B q}^{2}}
$$

For instance, an elliptical motion of the particle with the same vibration speed along $x$ and $y$ of $0.3 \mathrm{~m} / \mathrm{s}$ is simply obtained by setting the references $U_{L d}=$ $U_{B q}=0.3$ and $U_{L q}=U_{B d}=0$. The closed loop control is embedded into a DSP (STM32F405 from ST Microelectronics). The response time of the vibration speed for both modes is set to about $2 \mathrm{~ms}$ as presented in Fig. $3 \mathrm{~b}$ ).

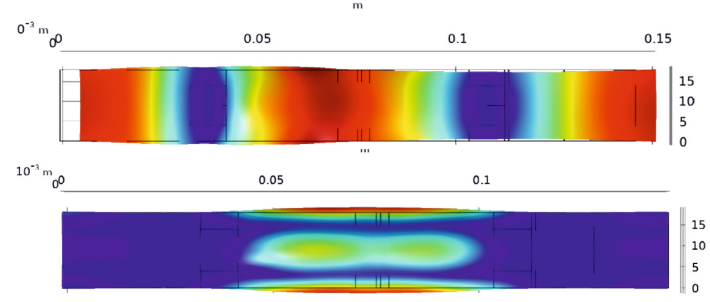

a)

(3)

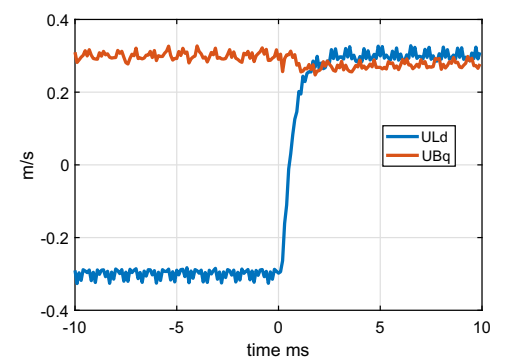

b)

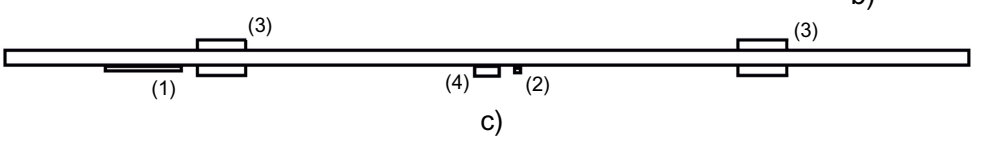

Fig. 3. Measurements on the device; a) deformation mode shape for the longitudinal mode (up) and the bending mode and b) transitory response of the vibration when $\Lambda$ is switched from -1 to 1 at $U_{B}=U_{L}=0.3 \mathrm{~m} / \mathrm{s}$. c) Side view of the aluminium plate. (1) longitudinal sensor, (2) bending sensor, (3) longitudinal actuator (4) bending actuator

\section{Materials and Methods}

\subsection{Participants}

Data were collected from seven healthy volunteers aged between 18 and 40 (3 females). Participants were wearing noise-cancelling headphones in order to prevent noise disturbance. All participants gave written informed consent. The investigation conformed to the principles of the Declaration of Helsinki and experiments were performed in accordance with relevant guidelines and regulations. 


\subsection{Experimental Set-Up}

The plate is mounted on a force sensor that measures the normal force at which a participant presses on the plate as shown in Fig. 2a). The keyclick rendering was performed as in [6]; two normal force thresholds were defined $f_{1}$ and $f_{2}=$ $f_{1}+0.3 N$. When the user reaches $f_{1}$, we turn on the two modes, with specific vibration speeds $U_{L}$ and $U_{B}$ leading to a value for $\Lambda$. When $f_{2}$ is reached, the direction of the elliptical motion is reversed simply by inverting $U_{B}$. At the end, when the user releases his finger from the surface, we turn off the device. The force is sampled at $10 \mathrm{kHz}$ by the DSP, and a Laptop PC is used to send the threshold value $f_{1}$ and the amplitude set points $U_{B}$ and $U_{L}$ to the DSP through USB.

\subsection{Experimental Procedure}

Before starting the experiment we ask the participants to wash their hands and we clean the plate to standardize the surface.

After a training period during when participants could discover the haptic feedback by testing the device at maximum intensity, at different $\Lambda$ and at two different activation force levels, they had to press once on the middle of the plate with their index finger as shown in Fig. 2. They were asked to say whether they could feel the virtual click or not. The estimation of the psycho-physical threshold was performed with a simple one-up one-down staircase procedure, which targets the $50 \%$ performance level on a psychometric function, corresponding to the level at which the probability of a detectable click equals the probability of an undetectable one [8].

As in [6], two force levels $f_{1}=[0.1 N, 0.4 N]$ are interleaved in order to avoid the prediction of the next stimulation intensity. Five different values for $\Lambda=[0.5$ 2/3 $11.52 \infty$ ] were tested. The order of the conditions was pseudo-randomized across participants to avoid learning curve effects. The experiment ended when 6 turnovers or 60 trials were achieved.

\section{Results}

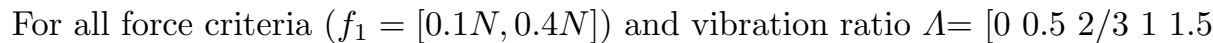
$2 \infty]$ we have computed the median level thresholds which are presented Fig. 4 and in Table 1.

The result shows very different threshold levels, depending on the value of $\Lambda$ : for $\Lambda \in[1 / 22 / 31]$, the threshold is close to $0.7 \mathrm{~m} / \mathrm{s}$, and doesn't change much with the force level. For $\Lambda \in\left[\begin{array}{ll}1.5 & 2 \infty\end{array}\right]$, the threshold level is higher (around 0.9) and decreases when the force level $f_{1}$ increases. Overall, the averaged threshold level is minimal for $\Lambda=1 / 2$ if $f_{1}=0.1 N$ and for $\Lambda=1$ when $f_{1}=0.4 N$. 
Table 1. Experimental results: $50 \%$ module threshold for different $\Lambda$ and different activation pressure levels

\begin{tabular}{|l|l|l|l|l|l|l|l|}
\hline \multicolumn{2}{|c|}{$\Lambda$} \\
\hline$f_{1}$ & & 0.5 & $2 / 3$ & 1 & 1.5 & 2 & $\infty$ \\
\hline \multirow{3}{*}{$0.1 \mathrm{~N}$} & Threshold (m.s-1) & 0.73 & 0.72 & 0.70 & 0.98 & 0.95 & 0.97 \\
\cline { 2 - 8 } & IQR & $0.62-0.77$ & $0.62-0.80$ & $0.65-0.73$ & $0.91-1.06$ & $0.62-0.97$ & $0.94-0.98$ \\
\cline { 2 - 8 } & var & 0.017 & 0.027 & 0.003 & 0.020 & 0.033 & 0.00092 \\
\hline \multirow{3}{*}{$0.4 \mathrm{~N}$} & Threshold (m.s-1) & 0.70 & 0.75 & 0.76 & 0.91 & 0.87 & 0.97 \\
\cline { 2 - 8 } & IQR & $0.56-0.84$ & $0.64-0.95$ & $0.68-0.77$ & $0.87-1.01$ & $0.68-1.02$ & $0.94-1$ \\
\cline { 2 - 8 } & var & 0.024 & 0.023 & 0.002 & 0.010 & 0.019 & 0.00071 \\
\hline
\end{tabular}

Interestingly, we also compare in Fig. 4c) and d) the variance for each condition. A F-Test $\left(\alpha=0.05, F_{\text {crit }}=4.28\right)$ have been performed on the variance of threshold level for $\Lambda=1$ against the others. The results are: for $f_{1}=0.1 N$ $\{\Lambda=0.5, \mathrm{~F}=4.68 ; \Lambda=2 / 3, \mathrm{~F}=7.34 ; \Lambda=1.5, \mathrm{~F}=5.5 ; \Lambda=2, \mathrm{~F}=8.83\}$ and for $f_{1}=0.4 N\{\Lambda=0.5, \mathrm{~F}=9.73 ; \Lambda=2 / 3, \mathrm{~F}=9.42 ; \Lambda=1.5, \mathrm{~F}=4.08$; $\Lambda=2, \mathrm{~F}=7.69\}$. We observe that for both force conditions, the condition $\Lambda=1$ produces less variance than other values.

\section{Discussion}

Our study shows that the elliptical motion of particles can indeed decrease the amount of vibration amplitude that is needed to give the illusion of a button click. Therefore, we can suggest that lateral displacement helps to increase the internal lateral stresses that are first stored when participants touch the plate, before they are released by inverting the direction of the particles' motion. Moreover, we have not seen many differences between the values for $\Lambda \leq 1$ on the detection threshold, and this threshold doesn't change with the force level; the condition $\Lambda=0$ has been tested but the averaged detection threshold has been found to be higher than the capability of the device. Therefore, we hypothesize that the value of $\Lambda$ does not change the lateral stresses when in the range $0.5 \leq \Lambda \leq 2 / 3$. We also show that the variance is minimal for $\Lambda=1$. Therefore, this gives rise to the optimal value at which the plate should operate. However, for now we do not give an explanation for this specific behaviour; a modelling that takes into account the intermittent contact could be used for that aim [13]. 


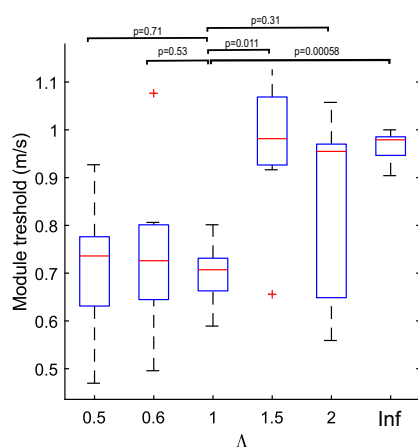

a)

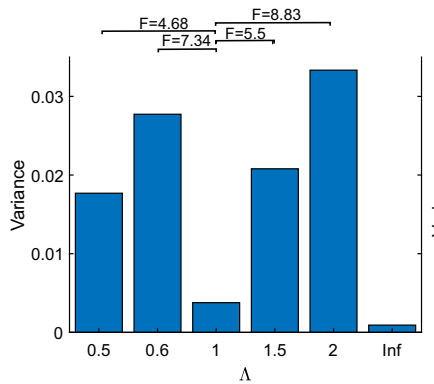

c)

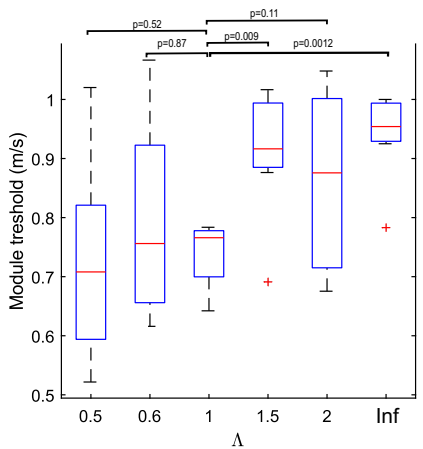

b)

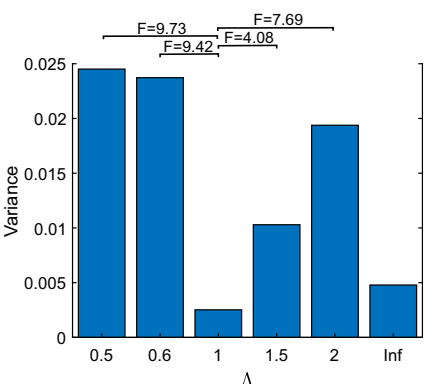

d)

Fig. 4. Experimental results: $50 \%$ module threshold, compute for different $\Lambda$ and different activation pressure levels : a) $f_{1}=0.4 N$, b) $f_{1}=0.1$. The error bars, the whisker boxes and the horizontal bars show respectively the min. and max. values, their interquartile range and the median value and corresponding variance $c$ ) and $d$ ) respectively.

\section{Conclusion}

In this paper we used two modes simultaneously to produce the simulation of a click on a static finger. To create a simulation with the lowest detection threshold as well as the lowest variance between participants, an equal amount of normal and lateral displacement should be set. This finding will be useful to design and control new tactile interfaces.

Acknowledgement. This work is supported by IRCICA (Research Institute on software and hardware devices for information and Advanced communication, USR CNRS $3380)$. 


\section{References}

1. Banter, B.: Touch screens and touch surfaces are enriched by haptic force-feedback. Inf. Display 26(3), 26-30 (2010)

2. Dai, X., Colgate, J.E., Peshkin, M.A.: Lateralpad: a surface-haptic device that produces lateral forces on a bare finger. In: 2012 IEEE Haptics Symposium (HAPTICS), pp. 7-4, March 2012

3. Emgin, S.E., Aghakhani, A., Sezgin, T.M., Basdogan, C.: Haptable: An interactive tabletop providing online haptic feedback for touch gestures. IEEE Trans. Vis. Comput. Graph. 25(9), 2749-2762 (2019)

4. Ghenna, S., Vezzoli, E., Giraud-Audine, C., Giraud, F., Amberg, M., LemaireSemail, B.: Enhancing variable friction tactile display using an ultrasonic travelling wave. IEEE Trans. Haptics 10(2), 296-301 (2017)

5. Giraud, F., Giraud-Audine, C.: Piezoelectric Actuators: Vector Control Method. Butterworth-Heinemann, Oxford (2019)

6. Gueorguiev, D., Kaci, A., Amberg, M., Giraud, F., Lemaire-Semail, B.: Travelling ultrasonic wave enhances keyclick sensation. In: Prattichizzo, D., Shinoda, H., Tan, H.Z., Ruffaldi, E., Frisoli, A. (eds.) EuroHaptics 2018. LNCS, vol. 10894, pp. 302312. Springer, Cham (2018). https://doi.org/10.1007/978-3-319-93399-3_27

7. Hudin, C., Panëels, S.: Localisation of vibrotactile stimuli with spatio-temporal inverse filtering. In: Prattichizzo, D., Shinoda, H., Tan, H.Z., Ruffaldi, E., Frisoli, A. (eds.) EuroHaptics 2018. LNCS, vol. 10894, pp. 338-350. Springer, Cham (2018). https://doi.org/10.1007/978-3-319-93399-3_30

8. Lekk, M.R.: Adaptive procedures in psychophysical research. Percept. Psychophys. 63, 1279-1292 (2001). https://doi.org/10.3758/BF03194543

9. Minikes, A., Bucher, I.: Noncontacting lateral transportation using gas squeeze film generated by flexural traveling waves-numerical analysis. J. Acoust. Soci. Am. 113(5), 2464-2473 (2003)

10. Monnoyer, J., Diaz, E., Bourdin, C., Wiertlewski, M.: Ultrasonic friction modulation while pressing induces a tactile feedback. In: Bello, F., Kajimoto, H., Visell, Y. (eds.) EuroHaptics 2016. LNCS, vol. 9774, pp. 171-179. Springer, Cham (2016). https://doi.org/10.1007/978-3-319-42321-0_16

11. Park, G., Choi, S., Hwang, K., Kim, S., Sa, J., Joung, M.: Tactile effect design and evaluation for virtual buttons on a mobile device touchscreen. In: Proceedings of the 13th MobileHCI conference, pp. 11-20 (2011)

12. Saleem, M.K., Yilmaz, C., Basdogan, C.: Psychophysical evaluation of change in friction on an ultrasonically-actuated touchscreen. IEEE Trans. Haptics 11(4), 599-610 (2018)

13. Torres Guzman, D.A., Lemaire-Semail, B., Kaci, A., Giraud, F., Amberg, M.: Comparison between normal and lateral vibration on surface haptic devices. In: 2019 IEEE World Haptics Conference (WHC), pp. 199-204, July 2019

14. Wallaschek, J.: Contact mechanics of piezoelectric ultrasonic motors. Smart Mater. Struct. 7(3), 369-381 (1998)

15. Xu, H., Klatzky, R.L., Peshkin, M.A., Colgate, J.E.: Localized rendering of button click sensation via active lateral force feedback. In: 2019 IEEE World Haptics Conference (WHC), pp. 509-514, July 2019 
Open Access This chapter is licensed under the terms of the Creative Commons Attribution 4.0 International License (http://creativecommons.org/licenses/by/4.0/), which permits use, sharing, adaptation, distribution and reproduction in any medium or format, as long as you give appropriate credit to the original author(s) and the source, provide a link to the Creative Commons license and indicate if changes were made.

The images or other third party material in this chapter are included in the chapter's Creative Commons license, unless indicated otherwise in a credit line to the material. If material is not included in the chapter's Creative Commons license and your intended use is not permitted by statutory regulation or exceeds the permitted use, you will need to obtain permission directly from the copyright holder. 